\title{
Does antiaggregant administration lead to early diagnosis in proximal colon cancer?
}

\author{
Ulas Aday, Ebubekir Gundes, Huseyin Ciyiltepe, Durmus Ali Cetin, Kamuran Cumhur Deger, \\ Selcuk Gulmez, Aziz Serkan Senger, Emre Bozdag \\ Department of Gastroenterology Surgery, Kosuyolu High Speciality Training and Research Hospital, Kartal, Istanbul, Turkey
}

\begin{abstract}
OBJECTIVE: Cancers of the proximal colon are often diagnosed in advanced stages with iron deficiency anemia and nonspecific symptoms. Aspirin and clopidogrel are commonly used antiaggregant agents for various clinical conditions. The aim of this study was to investigate the effects of antiaggregant medication on the early diagnosis of proximal colon cancer.
\end{abstract}

METHODS: Cases of colon cancer patients who had received curative surgical procedures between January 1 , 2013 and July 31, 2016 were retrospectively reviewed. The clinical and pathological results of patients who had used antiaggregant drugs were compared to those who had not.

RESULTS: During the studied period, 246 colorectal cancer patients underwent curative surgical procedures. Of the 67 patients with proximal colon cancer who were included in the study, 27 (40.3\%) had taken antiaggregant medication. The mean age of the antiaggregant group was 67.1 years (range: $34-88$ years), while it was 58.3 years (range: 34-83 years) for the non-antiaggregant group; the difference between the 2 groups was statistically significant $(p=0.03)$. A pathological evaluation revealed that $74.1 \%$ of the antiaggregant group was in the early stages (Stage I/II: $7 / 13$ ), while $42.5 \%$ of the non-antiaggregant group was in the early stages (Stage I/II: $2 / 15$ ); the difference was statistically significant $(p=0.011)$.

CONCLUSION: Antiaggregant medication has a positive effect on diagnosing proximal colon cancer at early stages. Patients using aspirin or clopidogrel should undergo a complete colonoscopic evaluation in the presence of gastrointestinal tract bleeding or newly developed anemia.

Keywords: Antiaggregant medication; early stage; proximal colon cancer.

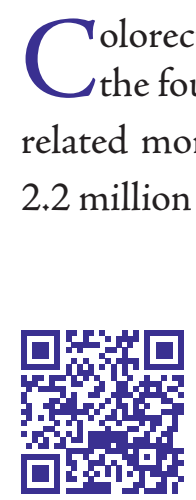

Received: February 02, 2017 Accepted: May 08, 2017 Online: August 26, 2017 
a screening tool has proven to be the gold standard of tumor detection because it enables the removal of premalignant lesions by polypectomy and renders pathological diagnosis possible [5].

Aspirin and clopidogrel are the antiaggregant agents most frequently administered to patients with, most notably, cardiac pathologies, or to those who have experienced cerebrovascular events or peripheral arterial diseases [6-8]. Gastrointestinal tract bleeding is one of the most significant side effects of antiaggregant use $[6,7]$. Lower gastrointestinal tract bleeding and the related anemia are prominent symptoms of colorectal cancer that enable early-stage diagnosis $[9,10]$. The increase in the prevalence of proximal colon cancer, aggressive behavior due to tumor biology, the failure to detect tumor in colonoscopy, and late diagnosis lead to an increase in mortality rates $[5,11,12]$.

This retrospective study compared the clinical and pathological results of proximal colon cancer (transverse and right colon) patients who had undergone curative surgical procedures based on whether antiaggregant agents had been administered. The effects of antiaggregant administration on proximal colon cancer staging were also evaluated.

\section{MATERIALS AND METHODS}

Cases of patients with colorectal cancer who had undergone curative surgical procedures at our clinic between January 1, 2013 and July 31, 2016 were retrospectively reviewed. Patients with proximal colon (cecum, ascending colon, hepatic flexura, or transverse colon-localized) tumors were included in the study, while those with rectum, splenic flexura, descending or sigmoid colon cancers were excluded. Moreover, patients who had surgical procedures for synchronous or metachronous tumors, who had been on anticoagulants, or who had received palliative interventions were excluded from the study. Patient data were collected from hospital records and final pathology results. Each patient's age, gender, indications for colonoscopy, antiaggregant use, and duration of hospitalization were recorded, in addition to the laboratory parameters analyzed at the time of diagnosis and the tumor, node, and metastasis (TNM) staging of the tumor.

Colonoscopy requests were divided into 2 groups: those that were related to bleeding conditions and those that were not. Bleeding-related conditions were defined as hematochezia, melena, positive fecal occult blood test (FOBT) results, and iron deficiency anemia. Conditions unrelated to bleeding were defined as abdominal pain, changes to defecation habits, weight loss, loss of appetite, obstructive findings, incidental findings by radiological evaluation, and findings resulting from colon cancer screening procedure. Anemia was defined as hemoglobin $(\mathrm{Hb})$ level lower than $12 \mathrm{~g} / \mathrm{dL}$ in $\mathrm{fe}$ males and lower than $13 \mathrm{~g} / \mathrm{dL}$ in males.

The pathological evaluation in this study was performed using the seventh edition of the American Joint Committee on Cancer TNM staging system [13]. Stages I and II were considered early stage, while stages III and IV were considered advanced stage. The duration of hospitalization was defined as the period from surgery to discharge, while early mortality was defined as mortality within the first 30 days after surgery. Study patients were divided into 2 groups: those who used antiaggregant agents (aspirin or clopidogrel) and those who did not. The clinical, laboratory and pathological results of each group were compared.

\section{Statistical analysis}

IBM SPSS Statistics for Windows, Version 21.0 (IBM Corp., Armonk, NY, USA) computer software was used for the statistical analyses of the data collected. Continuous variables were assessed using the Kolmogorov-Smirnov normality test. The data collected from the patients included in the study were given as mean, SD, and percentage, as appropriate. The Mann-Whitney $U$ test was used for non-normally distributed data, while the Student's t-test was performed for normally distributed data for comparisons between the groups. Categorical groups were compared with the chi-square test. A p-value of less than 0.05 was considered statistically significant. 
TABLE 1. Clinical characteristics of the patients

\begin{tabular}{lccc} 
& $\begin{array}{c}\text { Antiaggregant } \\
\text { Yes }(\mathrm{n}=27)\end{array}$ & $\begin{array}{c}\text { Antiaggregant } \\
\text { No }(\mathrm{n}=40)\end{array}$ & $0.03^{*}$ \\
\hline Age $($ Mean $\pm \mathrm{SD})$ & $67.1 \pm 11.1$ & $58.3 \pm 12.0$ & 0.80 \\
Sex (male/female) & $14 / 13$ & $22 / 18$ & 0.122 \\
Those with bleeding-related symptoms (\%) & $18(66.7 \%)$ & $19(47.5 \%)$ & - \\
Aspirin & 9 & - & 0.141 \\
Clopidogrel & 8 & - & \\
Dual therapy & 10 & $10.2 \pm 2.3$ & \\
Hb value at the time of diagnosis $(\mathrm{g} / \mathrm{dL} \pm \mathrm{SD})$ & $9.3 \pm 1.8$ & $16(28.1 \%)$ & 0.147 \\
Patients with anemia $(\mathrm{n}=57)$ & $12(21.1 \%)$ & $16(28.1 \%)$ & $9.2 \pm 3.7$ \\
$\quad$ Male $(\mathrm{Hb}<13 \mathrm{mg} / \mathrm{dL})$ & $13(22.8 \%)$ & $11.3 \pm 6.8$ & \\
$\quad$ Female $(\mathrm{Hb}<12 \mathrm{mg} / \mathrm{dL})$ & &
\end{tabular}

Hb: Hemoglobin; SD: Standard deviation. ${ }^{*} \mathrm{p}<0.05$.

\section{RESULTS}

Between January 1, 2013 and July 31, 2016, a total of 246 patients underwent curative colorectal cancer surgery at our clinic. Of these tumors, 77 (31.3\%) were localized in the rectum, 88 (35.8\%) in the distal colon, and $71(28.8 \%)$ in the proximal colon. Ten patients had synchronous or metachronous tumors. Of the patients with proximal colon cancer, 67 were included in the study. Three patients were excluded from the study due to warfarin use, while 1 was excluded due to missing data.

The average age of the patients was 61.8 years (range: $34-88$ years), and 31 patients $(46.3 \%)$ were female. Antiaggregant agents were administered to 27 patients $(40.3 \%)$ in the group; 40 patients $(59.7 \%)$ did not receive such treatment. The mean age of the antiaggregant and non-antiaggregant groups was 67.1 years (range: $34-88$ years) and 58.3 years (range: $34-83$ years), respectively; the difference between the groups was statistically significant $(p=0.03)$. While 8 of the antiaggregant patients used only clopidogrel and 9 used only aspirin, 10 used a combination of both. In both groups, colonoscopy request was most commonly caused by bleeding-related conditions; it was observed in $18(66.7 \%)$ antiaggregant group patients and in
19 (47.5\%) non-antiaggregant group patients. Abdominal pain was the second most common symptom in both groups; it was seen in 5 antiaggregant group patients and in 18 non-antiaggregant group patients. The mean $\mathrm{Hb}$ value at the time of diagnosis was $9.3 \pm 1.8 \mathrm{~g} / \mathrm{dL}$ in the antiaggregant group and $10.2 \pm 2.3 \mathrm{~g} / \mathrm{dL}$ in the non-antiaggregant group, but the difference was not found to be statistically significant $(p=0.14)$. The demographic, clinical, and laboratory characteristics and results of the 2 groups have been summarized in Table 1 .

Pathological evaluation revealed that 20 (74.1\%) patients in the antiaggregant group had early-stage cancer (Stage I/II: 7/13), while 17 (42.5\%) patients in the non-antiaggregant group had early-stage cancer (Stage I/II: 2/15); the difference between the groups was found to be statistically significant $(p=0.01)$. The mean number of excised lymph nodes in the antiaggregant group was 19.6 (range: 7-33 lymph nodes), while it was 24.9 (range: 11-59 lymph nodes) in the non-antiaggregant group, and the difference between the groups was statistically significant $(p=0.02)$. The mean number of positive lymph nodes was 0.55 (range: 0-7 lymph nodes) and 1.95 (range: 0-23 lymph nodes) in the antiaggregant and non-antiaggregant groups, respectively 
(Table 2). The difference between the 2 groups was found to be statistically significant $(p=0.006)$. The mean duration of hospitalization was 11.3 days (range: 6-37 days) in the antiaggregant group and 9.2 days (range: 5-20 days) in the non-antiaggregant group; there was no statistically significant difference between the groups ( $p=0.147)$.

The number of patients in the antiaggregant group receiving monotherapy (either aspirin or clopidogrel) was 17, while 10 patients received dual therapy (aspirin and clopidogrel) (Table 3). Neither had a statistically significant effect on early-stage diagnosis $(\mathrm{p}=0.201)$.

\section{DISCUSSION}

Colorectal cancers account for the third most common type of cancer in the world, and the rate of proximal colon cancer incidence has increased over the course of the last decades $[1,2,12,14]$. Proximal colon cancer has a poorer prognosis than left colon cancer because of the greater incidence of poor differentiation, tumors containing a mucinous component, late-stage diagnosis, and the advanced age of patients $[11,12,15]$. While anemia and non-specific symptoms are predominant in proximal colon cancer, symptoms related to changes in defecation habits, fresh rectal bleeding, mucoid discharge, and obstruction are more frequent in distal colon and rectum cancers [16]. Symptoms associated with hemorrhage (including melena, fresh rectal bleeding, iron deficiency anemia, and FOBT positivity) are witnessed in three-quarters of colorectal cancer patients. Early-stage diagnosis rates particularly increase when fresh rectal bleeding and melena are predominant $[17,18]$. However, diagnosis is usu-

TABLE2. Comparison of the pathological results between groups

Stage

Early stage (I/II)

Late stage (III/IV)

The number of excised lymph nodes (Mean \pm SD)

The number of metastatic lymph nodes [Median (Min.-Max.)]

SD: Standard deviation; Min: Minimum; Max: Maximum. ${ }^{*} p<0.05$

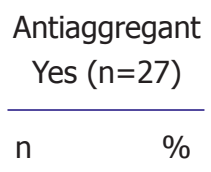

$$
\begin{aligned}
& \begin{array}{c}
\text { Antiaggregant } \\
\text { No }(n=40)
\end{array} \\
& \hline n \quad \%
\end{aligned}
$$

\begin{tabular}{|c|c|c|c|c|}
\hline 20 & 74.1 & 17 & 42.5 & $0.011 *$ \\
\hline 7 & 25.9 & 23 & 57.5 & \\
\hline \multicolumn{2}{|c|}{$19.6 \pm 6.3$} & \multicolumn{2}{|c|}{$24.9 \pm 9.5$} & $0.020 *$ \\
\hline \multicolumn{2}{|c|}{$0(0-7)$} & \multicolumn{2}{|c|}{$1(0-23)$} & $0.006 *$ \\
\hline
\end{tabular}

$\mathrm{p}$

TABLE 3. The effects of single or dual antiaggregant administration on staging

$\frac{\text { Early stage (I/II) } n=20}{\mathrm{n}}$

Antiaggregant treatment

Monotherapy

Dual therapy

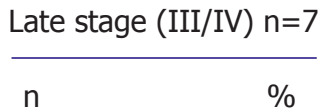


ally delayed and the survival rate is negatively affected by the presence of non-specific symptoms, such as iron deficiency anemia, abdominal pain, fatigue, and weight loss $[18,19]$. The inclusion of colonoscopy in the screening program has led to more early diagnoses of colorectal cancers and has enabled polypectomy of premalignant lesions, resulting in a significant reduction in mortality rates. Pathologies in the proximal colon can be missed because of contamination, incomplete colonoscopy, and the fact that the adenomas observed in the proximal colon are often sessile-flat type; this results in delayed diagnosis and impaired prognosis $[3,4,5,20]$.

Antiaggregant agents are frequently and increasingly administered to patients with cardiac pathologies, stroke, and peripheral arterial diseases, and they have proven to be significantly beneficial to patient outcomes. Bleeding in the gastrointestinal tract is one of the side effects of these agents. Antithrombotic agents, especially when used as dual therapy, cause rising FOBT positivity and may lead to early diagnosis of digestive system malignancies by increasing bleeding from polyps or tumors $[6,7]$. Gastrointestinal tract bleeding may be life-saving, as it often leads to early diagnosis, but it increases the false positivity rates for FOBT and causes an increase in negative colonoscopy rate $[6,9,21]$. In patients with cardiac and peripheral arterial disease, gastrointestinal system (especially colorectal) malignancies are more common than in the general population. This has been associated with accompanying characteristics like diabetes, obesity, and smoking, rather than antiaggregant agents [22].

Clopidogrel prevents platelets from sticking to each other and indirectly blocks angiogenesis and mucosal healing by binding to platelets' adenosine diphosphate receptors. This leads to the prolonged bleeding of polyps and ulcerated malignant lesions, beginning with the early stage [22, 23]. As with aspirin and warfarin, clopidogrel should be discontinued according to clinical suitability before the FOBT evaluation due to this bi-directional effect [24]. Bleeding caused by the use of aspirin is directly proportional to the dose administered. Most colorectal cancers develop in polyps. Aspirin reduces the rate of cancer incidence by inhibiting cyclo- oxygenase-2 (COX-2) in low doses (less than 300 mg IN) and COX-1 in high doses, thereby blocking a polyp's progression. Rothwell et al. [25] reported that long-term, regular low-dose aspirin intake reduced the incidence and mortality rates of colorectal cancer, which was more pronounced in right colon cancer cases.

Diagnosis is often delayed in the presence of anemia and nonspecific symptoms. In a study of 768 patients, Alexiusdottir et al. [16] reported that rectal bleeding and changes in bowel habits were more frequent in left colon tumors, which leads to earlierstage diagnosis. The authors also stated that anemia and other general symptoms were more frequent in right colon cancers leading to later-stage diagnosis [16]. Hreinsson et al. [17] reported a similar result: warfarin administration contributed to early-stage diagnosis by causing pronounced bleeding, whereas aspirin did not cause this effect [17].

Our center predominantly treats patients with cardiac and vascular pathologies. These pathologies are therefore present in a significant proportion of our patients who undergo surgical procedures of the gastrointestinal tract. This is also true of our patients who receive endoscopic and colonoscopic procedures. Twenty-seven (40.2\%) of 67 proximal colon cancer patients who underwent curative surgery and were included in this study were taking antiaggregant agents. In our country, colorectal cancer screening programs are not at a sufficient level to cover the entire population. Generally, colonoscopic evaluations are performed only in the presence of symptoms or within the screening schedule. In our study, bleeding-related symptoms and the resulting anemia were the most common reasons for colonoscopic evaluation in both groups. Although the distribution of symptoms was similar in both groups, we believe that the rate of early-stage diagnosis was higher in the antiaggregant group (Stage I/II, $74.1 \% ; p=0.011$ ) because those patients were regularly monitored by physicians due to their cardiac or vascular pathologies.

The prognoses and survival rates for cancers of the colon differ depending on the localization. The general view is that proximal colon cancer patients have a lower survival rate; however, there are several studies 
that document no difference in the survival rate for right colon cancer or that demonstrate a better rate $[8,12,14,20]$. Benedix et al. [20] reported in their 28,797-patient study that, although the rate of early diagnosis in descending and ascending colon cancers was higher than $50 \%$, the rate was lower in splenic flexura and cecal cancer. This may be explained in cecal cancer cases by prolonged stool transit time, prolonged stool exposure, a failure to detect tumor by colonoscopy, and a greater presence of poorly differentiated tumor types with aggressive prognosis; the reason is not clear in splenic flexura cancer cases [11, 20]. Weiss et al. [14] found that right colon cancer was more prevalent in the elderly and in female patients, and that advanced stage and poor differentiation characteristics were predominant. The authors, however, reported that there was no overall difference in 5-year mortality, but the mortality rate for stage II right colon cancer patients was found to be lower. In a study of 91,416 patients, Warschkow et al. [12] reported survival rate of right and left cancers following a mean follow-up of 38 months. Their results revealed that the prognosis and survival rate were better for stage I and II right colon cancer than for those of left colon cancer, while they proved to be similar for both in stage III. The authors' study opened up new discussions about the issue.

There are limitations to our work, including its retrospective structure, the limited number of patients, the absence of an evaluation of survival rate, a lack of comparison with the distal colon, and the lack of comparison between different localizations of the proximal colon. However, this study allows for the comparison of those who use antiaggregant agents with those who do not by forming a homogeneous group of proximal colon cancer cases.

\section{Conclusion}

The frequency of the administration of antiaggregant agents has been on the rise. Anemia due to gastrointestinal tract bleeding caused by these agents may be a sign of early-stage proximal colon cancer. The use of aspirin or clopidogrel has a positive effect on early-stage diagnosis of proximal colon cancer. Concerned clinicians should pay careful attention to newly diagnosed anemia in patients undergoing antiaggregant treatment, and patients should receive colonoscopic and endoscopic evaluations.

Conflict of Interest: None declared.

Financial Disclosure: The authors declared that this study has received no financial support.

Authorship contributions: Concept - U.A., E.G., H.C., D.A.C.; Design - U.A., E.G., D.A.C., E.B.; Supervision - K.C.D., S.G., E.B., A.S.S.; Materials - A.S.S., S.G., E.B.; Data collection \&/or processing - U.A., E.G., D.A.C., H.C., E.B., A.S.S., S.G., KCD; Analysis and/or interpretation - U.A., E.G., K.C.D., E.M.; Literature search - U.A., E.G., H.C., D.A.C., S.G., E.B.; Writing - U.A., E.G., H.C; Critical review - E.B., K.C.D., S.G.

\section{REFERENCES}

1. Arnold M, Sierra MS, Laversanne M, Soerjomataram I, Jemal A, Bray F. Global patterns and trends in colorectal cancer incidence and mortality. Gut 2017;66:683-91.

2. Favoriti P, Carbone G, Greco M, Pirozzi F, Pirozzi RE, Corcione F. Worldwide burden of colorectal cancer: a review. Updates Surg 2016;68:7-11.

3. Jacob BJ, Moineddin R, Sutradhar R, Baxter NN, Urbach DR. Effect of colonoscopy on colorectal cancer incidence and mortality: an instrumental variable analysis. Gastrointest Endosc 2012;76:355-64.e1.

4. Pan J, Xin L, Ma YF, Hu LH, Li ZS. Colonoscopy Reduces Colorectal Cancer Incidence and Mortality in Patients With Non-Malignant Findings: A Meta-Analysis. Am J Gastroenterol 2016;111:355-65.

5. Matsuda T, Ono A, Kakugawa Y, Matsumoto M, Saito Y. Impact of screening colonoscopy on outcomes in colorectal cancer. Jpn J Clin Oncol 2015;45:900-5.

6. Ishizaka N, Sakamoto A, Fujishiro M, Nagai R, Koike K. Gastrointestinal malignancies and cardiovascular diseases-non-negligible comorbidity in an era of multi-antithrombotic drug use. J Cardiol 2011;58:199-207.

7. Sakamoto A, Fujishiro M, Koike K, Nagai R, Ishizaka N. The prevalence of malignant neoplastic and non-malignant gastrointestinal lesions in cardiology inpatients. J Cardiol 2013;61:181-5.

8. Kirshtein B, Ariad S, Mizrahi S, Man S, Walfisch S. Rectal bleeding and previous anticoagulant treatment in patients with colorectal cancer do not predict outcome. Tech Coloproctol 2007;11:121-6.

9. Norton SA, Armstrong CP. Lower gastrointestinal bleeding during anticoagulant therapy: a life-saving complication? Ann R Coll Surg Engl 1997;79:38-9.

10. Symeonidis D, Koukoulis G, Christodoulidis G, Mamaloudis I, Chatzinikolaou I, Tepetes K. Impact of antiplatelet treatment on colorectal cancer staging characteristics. World J Gastrointest Endosc 2012;4:409-13.

11. Benedix F, Meyer F, Kube R, Gastinger I, Lippert H. Right-and 
left-sided colonic cancer - different tumour entities. Zentralbl Chir 2010;135:312-7.

12. Warschkow R, Sulz MC, Marti L, Tarantino I, Schmied BM, Cerny $\mathrm{T}$, et al. Better survival in right-sided versus left-sided stage I - III colon cancer patients. BMC Cancer 2016;16:554.

13. Edge S, Byrd DR, Compton CC, Fritz AG, Greene F, Trotti A. AJCC Cancer Staging Manual. $7^{\text {th }}$ ed. New York: Springer-Verlag; 2010.

14. Weiss JM, Pfau PR, O'Connor ES, King J, LoConte N, Kennedy $\mathrm{G}$, et al. Mortality by stage for right-versus left-sided colon cancer: analysis of surveillance, epidemiology, and end resultsMedicare data. J Clin Oncol 2011;29:4401-9.

15. Powell AG, Wallace R, McKee RF, Anderson JH, Going JJ, Edwards J, et al. The relationship between tumour site, clinicopathological characteristics and cancer-specific survival in patients undergoing surgery for colorectal cancer. Colorectal Dis 2012;14:1493-9.

16. Alexiusdottir KK, Möller PH, Snaebjornsson P, Jonasson L, Olafsdottir EJ, Björnsson ES, et al. Association of symptoms of colon cancer patients with tumor location and TNM tumor stage. Scand J Gastroenterol 2012;47:795-801.

17. Hreinsson JP, Jonasson JG, Bjornsson ES. Bleeding-related symptoms in colorectal cancer: a 4-year nationwide populationbased study. Aliment Pharmacol Ther 2014;39:77-84.

18. Thompson MR, Asiimwe A, Flashman K, Tsavellas G. Is earlier referral and investigation of bowel cancer patients presenting with rectal bleeding associated with better survival? Colorectal
Dis 2011;13:1242-8.

19. Alexiusdottir KK, Snaebjornsson P, Tryggvadottir L, Jonasson L, Olafsdottir EJ, Björnsson ES, et al. Colon cancer: association of histopathological parameters and patients' survival with clinical presentation. APMIS 2013;121:901-7.

20. Benedix F, Schmidt U, Mroczkowski P, Gastinger I, Lippert H, Kube R; Study Group Colon/Rectum Carcinoma (Primary Tumor). Colon carcinoma-classification into right and left sided cancer or according to colonic subsite?-Analysis of 29,568 patients. Eur J Surg Oncol 2011;37:134-9.

21. Johannsdottir GA, Onundarson PT, Gudmundsdottir BR, Bjornsson ES. Screening for anemia in patients on warfarin $\mathrm{fa}$ cilitates diagnosis of gastrointestinal malignancies and pre-malignant lesions. Thromb Res 2012;130:e20-5.

22. Chan AO, Jim MH, Lam KF, Morris JS, Siu DC, Tong T, et al. Prevalence of colorectal neoplasm among patients with newly diagnosed coronary artery disease. JAMA 2007;298:1412-9.

23. Cryer B. Reducing the risks of gastrointestinal bleeding with antiplatelet therapies. N Engl J Med 2005;352:287-9.

24. Sawhney MS, McDougall H, Nelson DB, Bond JH. Fecal occult blood test in patients on low-dose aspirin, warfarin, clopidogrel, or non-steroidal anti-inflammatory drugs. Dig Dis Sci 2010;55:1637-42.

25. Rothwell PM, Wilson M, Elwin CE, Norrving B, Algra A, Warlow CP, et al. Long-term effect of aspirin on colorectal cancer incidence and mortality: 20-year follow-up of five randomised trials. Lancet 2010;376:1741-50. 